\title{
Prostaglandin E2 triggers cytochrome P450 17 $\alpha$ hydroxylase overexpression via signal transducer and activator of transcription 3 phosphorylation and promotes invasion in endometrial cancer
}

\author{
JIEQI KE, ZHEN SHEN, MIN LI, CHENG PENG, PING XU, MEIMEI WANG, \\ YI ZHU, XUEFEN ZHANG and DABAO WU
}

Department of Obstetrics and Gynecology, Anhui Provincial Hospital, Hefei, Anhui 230001, P.R. China

Received October 11, 2016; Accepted September 22, 2017

DOI: $10.3892 / \mathrm{ol} .2018 .9165$

\begin{abstract}
Prostaglandin E2 (PGE2) is the most common prostaglandin in the human body, meaning that its malfunction impacts on the development of numerous diseases. Prostaglandin E synthase 2 (PTGES2) is involved in the synthesis of PGE2. In the present study, immunohistochemistry of PTGES2 was performed in 152 patients with endometrial cancer and in 66 patients with normal endometria. The results indicate a notable association among increased expression of PTGES2 and age $(\mathrm{P}=0.0092)$ and the depth of myometrial invasion $(\mathrm{P}<0.0001)$. Reverse transcription-quantitative polymerase chain reaction and western blot analysis demonstrated that cytochrome P450 17 $\alpha$ hydroxylase (CYP17), an enzyme for androgen synthesis, is overexpressed following PGE2 stimulation via signal transducer and activator of transcription 3 (STAT3) phosphorylation. ELISA also detected increased androgen (testosterone) secretion. Further invasion of endometrial cancer cells was induced at high androgen levels and when CYP17 was overexpressed. Furthermore, the present study observed that CYP17 is overexpressed via STAT3 phosphorylation in endometrial cancer cells, which grow at a high concentration of PGE2, resulting in increased androgen secretion. Concentrations of estrogen and progesterone were not elevated, while the concentration of androgens was. Overall, a high concentration of androgens caused increased invasion of endometrial cancer cells. A high concentration of androgens, which is initiated by a high expression of PTGES2 and a high concentration of PGE2, is an important promoter of myometrial invasion in endometrial cancer.
\end{abstract}

Correspondence to: Professor Dabao Wu, Department of Obstetrics and Gynecology, Anhui Provincial Hospital, 17 Lu Jiang Road, Hefei, Anhui 230001, P.R. China

E-mail:wudabao5907@126.com

Key words: prostaglandin E2, signal transducer and activator of transcription 3, cytochrome P450 17 $\alpha$ hydroxylase, endometrial cancer

\section{Introduction}

Endometrial cancer is the most common reproductive cancer in women (1). In China, the incidence of endometrial cancer is 7.44 cases per 1,000,000 individuals (2), and in the USA, the disease causes 8,590 mortalities every year (3).

Prostaglandin E2 (PGE2) is the most common prostaglandin in humans and was first reported in 1936 (4). A previous study has revealed that PGE2 has a significant function in reproductive, neuronal, metabolic and immune systems (5). In two previous studies by Che et al (6) and Zhu et al $(6,7)$, certain cytokines, such as interleukin (IL)-6 and oncostatin $\mathrm{M}$, were revealed to promote proliferation and invasion of endometrial cancer cells. Certain studies have also reported that PGE2 serves a role in the development of ovarian, breast and colorectal cancer (8-10). However, to the best of our knowledge, no studies have investigated the mechanism of the role served by PGE2 in endometrial cancer.

The signal transducer and activator of transcription 3 (STAT3) transcription factor was identified as a downstream effecter of inflammatory mediators that modulates gene expression and metabolism (11). STAT3 underwent phosphorylation and activated the expression of numerous STAT3-regulated genes (12). STAT3 is usually highly expressed in cancer cells, and high levels of STAT3 prompt a poor outcome in ovarian cancer, glioblastoma, breast cancer and prostate cancer (13-17).

The cytochrome P450 (CYP) superfamily of enzymes mediates the catalytic conversion of drugs to reactive products that bind to macromolecules, including proteins and DNA. CYP enzymes account for $\sim 75 \%$ of total drug metabolism (18). CYP17 $\alpha$ hydroxylase (CYP17), an enzyme involved in androgen synthesis, has been implicated in the pathogenesis of numerous cancer types (19). A previous study has observed that inhibiting CYP17 is beneficial in prostate carcinoma (20), suggesting that CYP17 may be an important factor in hormone-associated cancer types.

In the present study, the PGE2 synthase-promoted expression of a P450 enzyme in endometrial cancer cells was analyzed, and the results identified CYP17 as the main enzyme involved in this process. These factors resulted in enhanced invasion in endometrial cancer cells. 


\section{Materials and methods}

Reagents and antibodies. PGE2 (cat. no. 363-24-6) and androgen (cat. no. 1424-00-6) were purchased from Sigma-Aldrich (Merck KGaA, Darmstadt, Germany). JSI-124 (cat. no. 2222-07-3) was obtained from Santa Cruz Biotechnology, Inc. (Dallas, TX, USA). Prostaglandin E synthase 2 (PTGES2) antibody (cat. no. 10881-1-AP, dilution at 1:1,000) was purchased from Protein Tech Group, Inc. (Chicago, IL, USA). Antibodies against CYP17 (cat. no. ab125022, dilution at 1:2,000), CYP19 (cat. no. ab35604, dilution at 1:1,000), IL-1 (cat. no. ab8320, dilution at 1:2,000), pSTAT3 (phosphorylated Y705, cat. no. ab76315, dilution at 1:20,000 and phosphorylated S727, cat. no. ab32143, dilution at 1:2,000) were purchased from Abcam (Cambridge, UK). The ELISA kits for estrogen (cat. no. CEA461Ge, 96T), androgen (testosterone, cat. no. CEA458Ge, 96T) and progesterone (cat. no. CEA459Ge, 96T) were purchased from Cloud-Clone Corp. (Houston, TX, USA).

Patients and samples. Tissue samples for immunohistochemistry (IHC) were obtained from 152 patients (all females aged between 34 and 79 years, with a mean age of 53.6 years) with endometrial cancer who had undergone surgical resection at Anhui Provincial Hospital (Hefei, China) between 2010 and 2016, and from 66 patients with normal endometria during curettage of the uterus. Clinicopathological characteristics including age, FIGO stage and grade (21), myometrial invasion and nodal metastasis were included. The project was approved by the Human Investigation Ethics Committee of Anhui Provincial Hospital and informed consent was obtained from all patients prior to the study.

Cell lines and culture conditions. The human endometrial cancer Ishikawa cell line, the $293 \mathrm{~T}$ cell line and primary endometrial (PE) cells were obtained from Dr Feizhou Jiang (Department of Obstetrics and Gynecology, The First Affiliated Hospital of Soochow University, Suzhou, China). Ishikawa, PE and 293T cells were grown in Dulbecco's modified Eagle's medium (DMEM)/F-12 (Gibco; Thermo Fisher Scientific, Inc., Waltham, MA, USA) supplemented with $10 \%$ fetal bovine serum (Gibco; Thermo Fisher Scientific, Inc.). Ishikawa, PE and $293 \mathrm{~T}$ cells were incubated at $37^{\circ} \mathrm{C}$ in a humidified atmosphere containing $5 \% \mathrm{CO}_{2}$. All experiments were performed at the third passage following thawing.

Total RNA extraction, reverse transcription-quantitative polymerase chain reaction $(R T-q P C R)$. Total RNA was isolated from Ishikawa cells using TRIzol reagent (cat no. 15596-026; Invitrogen; Thermo Fisher Scientific, Inc.) and cDNA was prepared using the reverse transcriptase kit (Takara Biotechnology Co., Ltd., Dalian, China). RT-qPCR was conducted using an ABI Prism 7500 sequence detection system (Applied Biosystems; Thermo Fisher Scientific, Inc.) and performed with the SYBR Green PCR Master mix (Toyobo Life Science, Osaka, Japan). According to supplier's protocol, the PCR conditions comprised $95^{\circ} \mathrm{C}$ for $30 \mathrm{sec}$, followed by 40 cycles of amplification $\left(95^{\circ} \mathrm{C}\right.$ for $3 \mathrm{sec}$ and $60^{\circ} \mathrm{C}$ for $30 \mathrm{sec}$ ). The $2^{-\Delta \Delta \mathrm{Cq}}$ method was used to analyze the relative changes in gene expression (22). The results were expressed relative to the internal reference gene GAPDH. Sequences of the primer pairs used are listed in Table I.

Western blot analysis. For western blot analysis, cells were lysed in cell lysis buffer (Beyotime Institute of Biotechnology, Nantong, China) for $30 \mathrm{~min}$ at $4^{\circ} \mathrm{C}$. Total protein, determined using a bicinchoninic acid assay (cat. no. P0010S; Beyotime Institute of Biotechnology). A total of $15 \mu \mathrm{g}$ protein per lane, was fractionated using SDS-PAGE (10\% gel) and was transferred onto polyvinylidene fluoride membranes, blocking with 5\% skim milk which dissolved in DMEM for $2 \mathrm{~h}$. The membranes were then incubated with the appropriate aforementioned primary antibodies (IL-1, CYP17, CYP19, pSTAT3-S727 and pSTAT3-Y705) for $24 \mathrm{~h}$ in $4^{\circ} \mathrm{C}$, followed by incubation with horseradish peroxidase-conjugated secondary antibody (cat. no. sc-280786, dilution at 1:10,000 for $2 \mathrm{~h}$ in $4^{\circ} \mathrm{C}$, Santa Cruz Biotechnology, Inc.). Following three further washes in TBS, the proteins were detected and visualized using an electrochemiluminescence system (Pierce; Thermo Fisher Scientific, Inc.) and GAPDH (cat. no. ab184531; dilution at 1:10,000; Abcam) was used as an internal control.

IHC. The tissue sections were initially fixed in $10 \%$ formalin solution at $4^{\circ} \mathrm{C}$ for 2 days and paraffin embedded. The tissue sections were subsequently subjected to microtome sectioning (5 $\mu \mathrm{m})$. The sections were placed on glass slides and immersed with $100 \%$ ethanol (5 min) and boiling water $(30 \mathrm{~min})$ three times. The endogenous peroxidase activity was blocked by immersing the sections in freshly prepared $10 \% \mathrm{H}_{2} \mathrm{O}_{2}$ and $10 \%$ methanol in $1 \mathrm{X}$ PBS for $20 \mathrm{~min}$. The sections underwent trypsin treatment $\left(0.1 \%\right.$ trypsin in $\left.0.1 \% \mathrm{CaCl}_{2}\right)$ for $10 \mathrm{~min}$ to cleave the protein crosslinks to assess the antigen and epitope. Nonspecific antigens were blocked using 4\% bovine serum albumin (BSA; Sigma-Aldrich; Merck KGaA, Darmstadt, Germany) for $2 \mathrm{~h}$ at room temperature. The membranes were incubated with anti-PTGES2 (1:100; ProteinTech Group, Inc.) overnight at $4^{\circ} \mathrm{C}$. Following incubation, the sections were thoroughly washed with $1 \mathrm{X}$ PBS and incubated with a goat anti-rabbit secondary antibody (dilution, 1:3,000; cat no. ab6721; Abcam) for $1 \mathrm{~h}$ at room temperature. Following washing to prevent non-specific binding, the sections were stained with diaminobenzidine (DAB; cat no. ab64238; Abcam). The percentage of positively stained cells was rated as follows: 0 points, $0 \%$; 1 point, $1-25 \%$; 2 points, 26-50\%; 3 points, $51-75 \%$; and 4 points, $\geq 75 \%$. The staining intensity was rated in the following manner: 0 points, negative staining; 1 point, weak intensity; 2 points, moderate intensity; and 3 points, strong intensity. Subsequently, immunoreactivity scores for each case were obtained by multiplying the values of the two parameters described above. The average score for all 5 random fields at x200 magnification was used as the histological score (HS). Tumors were categorized into 2 groups based upon the HS: The low-expression group (HS, 0-6) and the high-expression group (HS, 7-12).

Transwell invasion assays. For Transwell invasion assays, the upper side of an $8-\mu \mathrm{m}$ pore, $6.5-\mathrm{mm}$ polycarbonate Transwell filter (Corning Incorporated, Corning, NY, USA) chamber was uniformly coated with Matrigel basement membrane matrix (BD Biosciences, Bedford, MA, USA) for $2 \mathrm{~h}$ at $37^{\circ} \mathrm{C}$ prior 
Table I. Primer sequences for quantitative polymerase chain reaction analysis.

\begin{tabular}{lll}
\hline Gene & \multicolumn{1}{c}{ Forward $\left(5^{\prime}-3^{\prime}\right)$} & \multicolumn{1}{c}{ Reverse $\left(5^{\prime}-3^{\prime}\right)$} \\
\hline PTGES2 & CTTCCTTTTCCTGGGCTTCG & GAAGACCAGGAAGTGCATCCA \\
GAPDH & GAAGGTGAAGGTCGGAGTC & GAAGATGGTGATGGGATTTC \\
TNF- $\alpha$ & TGGCCTCCCTCTCATCAGTT & ATCGGCTGGCACCACTAGTT \\
CYP19 & TGGAAATGCTGAACCCGATAC & AATTCCCATGCAGTAGCCAGG \\
CYP17 & AGAATTCTCTGGTCGGCC & TTCTCCAGTTTCTGGCCA \\
IL-1 & GCCCTAAACAGATGAAGTGCTC & GAACCAGCATCTTCCTCAG \\
IL-10 & GGCACCCAGTCTGAGAACAG & ACTCTGCTGAAGGCATCTCG
\end{tabular}

PTGES2, prostaglandin E synthase 2; TNF- $\alpha$, tumor necrosis factor- $\alpha$; CYP, cytochrome P450; IL, interleukin; siCYP17, cytochrome P450 $17 \alpha$ hydroxylase small interfering RNA.

to the cells being added. A total of $2 \times 10^{4}$ Ishikawa cells were seeded in serum-free DMEM/F-12 into the upper chamber of a Transwell filter (in triplicate) and were incubated in $37^{\circ} \mathrm{C}$ for $48 \mathrm{~h}$. Invasive cells, which had reached the lower chamber, which contained DMEM/F-12 supplemented with 10\% FBS, were fixed in $4 \%$ paraformaldehyde, stained in $0.5 \%$ crystal violet (Beyotime, $20^{\circ} \mathrm{C}, 10 \mathrm{~min}$ ) and counted using a confocal microscope (Olympus, Shibuya, Japan). A total of 5 fields was counted for each Transwell filter. Each field was counted and images were captured at x200 magnification.

Transfection. Control groups and siCYP17 groups were created. Ishikawa cells with DMEM medium only was used as the control group. To inhibit the expression of the target gene, high-performance liquid chromatography-purified small interfering CYP17 siRNAs (1 $\mu 1$ in $50 \mu 1$ DMEM medium; 5'-3': GCUGGAGAAGAUCAUUUGU,) were prepared according to the sequence of the target gene. A scrambled siRNA with no homology to any known human mRNA was used as a negative control (siCo). siRNA oligonucleotide duplexes were synthesized by Shanghai GenePharma Co., Ltd. (Shanghai, China). The sequences of the siRNA oligonucleotides are provided in Table I. Cells were seeded onto 6-well plates at 70-80\% confluence and grown overnight prior to transfection. Transfection of the cells with the siRNA or siCo was accomplished using the Lipofectamine 2000 transfection reagent (Invitrogen; Thermo Fisher Scientific, Inc.) according to the manufacturer's protocols in $48 \mathrm{~h}$.

Plasmids. Flag-CYP17 was subcloned into a PiggyBac vector (Shanghai GenePharma Co., Ltd.) and transfected with a help vector. To obtain a stable and pure CYP17-overexpressing (CYP17 OE) cell population, selection with $300 \mu \mathrm{g} / \mathrm{ml}$ hygromycin (Santa Cruz Biotechnology, Inc.) was performed in $37^{\circ} \mathrm{C}$ until the control cells died. For the immunofluorescence staining, CYP17-Flag was subcloned to the pLenti-GIII-CM V-IRES-puro-SV40-GFP vector (Shanghai GenePharma Co., Ltd.). To obtain a pure cell population, $2 \mu \mathrm{g} / \mathrm{ml}$ puromycin (Santa Cruz Biotechnology, Inc.) selection was performed after $24 \mathrm{~h}$ of transfection at $37^{\circ} \mathrm{C}$. The DMEM/F12 medium was then changed after $12 \mathrm{~h}$ when the blank control cells died. All the cells were visually GFP-positive under a fluorescence microscope. CYP17 plasmids were obtained from Shanghai
GenePharma Co., Ltd. The plasmids were transfected with Lipofectamine 3000 into Ishikawa cells according to the manufacturer's protocols.

ELISA. Concentrations of the estrogen, androgen and progesterone hormones were detected in culture medium of PE cells and Ishikawa cells using solid phase sandwich ELISA according to the manufacturer's protocols (Cloud-Clone Corp.). The hormone assay sensitivity was $0.1 \mathrm{pg} / \mathrm{ml}$ and the assay range was $1.03-20,000 \mathrm{pg} / \mathrm{ml}$. For statistical analysis, culture medium was independently collected three times. The ELISA kits used were as follows: ELISA kit for Estradiol (estrogen, cat. no. CEA461Ge, 96T), ELISA kit for Testosterone (cat. no. CEA458Ge, 96T) and ELISA kit for Progesterone (cat. no. CEA459Ge, 96T), all purchased from Cloud-Clone Corp.

Statistical analysis. All quantitative data are presented as the mean \pm standard deviation. Data were analyzed using one-way analysis of variance (ANOVA) with a least significant difference test. All statistical analyses were performed using SPSS 17.0 (SPSS, Inc., Chicago, IL, USA). P<0.05 was considered to indicate a statistically significant difference. All experiments were performed $\geq 3$ times.

\section{Results}

PTGES2 expression is increased in endometrial cancer. PTGES2 is involved in the synthesis of PGE2. Recent studies have indicated that PGE2 may be a mitogen with a role in a number of cancer types (8-10). IHC was performed in normal endometria and endometrial cancer tissues. Compared with that in the normal endometria, the expression of PTGES2 was significantly upregulated in the endometrial cancer tissues (Fig. 1A and B; Table II). Statistical analysis revealed that increased expression of PTGES2 was significantly associated with the age of the patient $(\mathrm{P}=0.0092)$ and the depth of myometrial invasion $(\mathrm{P}<0.0001)$, but not with any other characteristics (FIGO stage, grade and nodal metastasis, Table III). As PTGES2 is required for the synthesis of PGE2, an ELISA was performed to determine the PGE2 concentration in Ishikawa cells, a typical endometrial cancer cell line. A significantly higher concentration of PGE2 was observed in these cells compared with that observed in the PE cells and 
Table II. Expression of PTGES2 in normal endometria and endometrial cancer.

HS of PTGES2, $n$

\begin{tabular}{lcccc}
\cline { 3 - 4 } Groups & Patients, $n$ & $\begin{array}{c}\text { Low expression } \\
\text { group }(\mathrm{HS}<6)\end{array}$ & $\begin{array}{c}\text { High expression } \\
\text { group }(\mathrm{HS} \geq 6)\end{array}$ & P-value \\
\hline Normal endometria & 66 & 49 & 17 & $<0.001^{\mathrm{a}}$ \\
Endometrial cancer & 152 & 56 & 96 & \\
\hline
\end{tabular}

${ }^{\mathrm{a}} \mathrm{P}<0.05$, determined using the $\chi^{2}$ test. PTGES2, prostaglandin E synthase 2; HS, histological score.
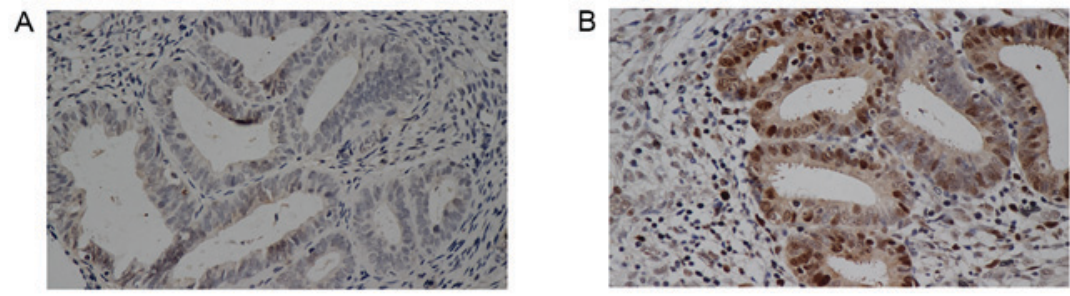

C

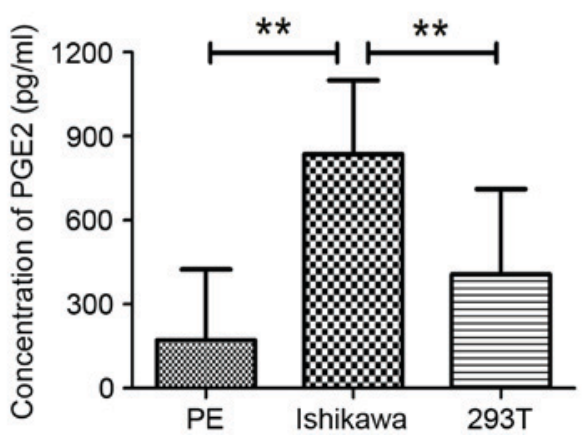

Figure 1. PTGES2 expression in different endometrial cells and PGE2 concentration in different cell types. Representative micrographs of PTGES2 staining in (A) endometrium and (B) endometrial cancer tissues (magnification, x400). (C) ELISA for PGE2 concentration in PE cells, Ishikawa cells and 293T cells. All cell types were cultured for $48 \mathrm{~h} .{ }^{* *} \mathrm{P}<0.01$, analyzed by one-way analysis of variance. PTGES2, prostaglandin E synthase 2; PGE2, prostaglandin E2; PE, primary endometrial.

the 293T cells (Fig. 1C). This confirmed that endometrial cells grow in a high concentration of PGE2. Therefore, the Ishikawa cells were selected for further study.

PGE2 promotes CYP17 expression in endometrial cancer cells via STAT3. RT-qPCR was performed to test the expression of certain potential mRNA targets identified in previous studies (23-27). As the results indicated, CYP17 expression was significantly increased following PGE2 stimulation, whereas the differences among other cytokines were not significant (Fig. 2A). Subsequent western blot analysis was performed to investigate CYP17 expression changes following PGE2 stimulation (Fig. 2B). As with the RT-qPCR results, CYP17 expression increased mainly in these reported targets. In a previous study, prostaglandin E2 receptor 4 (EP4) was identified as the receptor of PGE2 (28). As EP4 is not a direct DNA promoter, it is unable to directly stimulate CYP17 expression. Subsequently, phosphorylation of the DNA binding protein, STAT3, was examined in Ishikawa cells. The results demonstrated that two STAT3 phosphorylated residues at S727 and Y705 exhibited increased CYP17 expression in
PGE2-stimulated Ishikawa cells. However, this increase was more notable at S727 than at Y705 and the increase of CYP17 was prevented by the presence of JSI-124, an inhibitor of phosphorylation (Fig. 2C). These results suggest that STAT3 may be a downstream protein of PGE2 and that PGE2 increases CYP17 expression.

High expression of CYP17 promotes androgen secretion and invasion in endometrial cancer cells. CYP17 is known to be the key enzyme involved in the synthesis of sex hormones. The concentrations of 3 hormones, estrogen, androgen (testosterone) and progesterone, in Ishikawa cells, were determined using ELISA. The results indicated that the concentration of androgen in the Ishikawa cells was more elevated than the concentrations of the other 2 hormones, compared with that in the PE cells or the control group (Fig. 3A). For further study, Ishikawa cells inhibited with CYP17 siRNA (siCYP17) and others overexpressing CYP17 (CYP17 OE) were prepared (Fig. 3B-D). CYP17 OE Ishikawa cells had been transfected with Flag-CYP17 plasmids, and exhibited an increased androgen concentration (Fig. 3E). Subsequently, 
Table III. Associations between PTGES2 expression and clinicopathological characteristics in endometrial cancer.

\section{HS of PTGES2, n}

Characteristics

Patients, $\mathrm{n}$

\section{Low expression}

group $(\mathrm{HS}<6)$
High expression group (HS $\geq 6$ )
P-value

\begin{tabular}{|c|c|c|c|c|}
\hline Total & 152 & 56 & 96 & \\
\hline \multicolumn{5}{|l|}{ Age, years } \\
\hline$\geq 55$ & 78 & 21 & 57 & \multirow[t]{2}{*}{$0.0092^{a}$} \\
\hline$<55$ & 74 & 35 & 39 & \\
\hline \multicolumn{5}{|l|}{ FIGO stage } \\
\hline Stage I-II & 126 & 48 & 78 & \multirow[t]{2}{*}{0.4804} \\
\hline Stage III-IV & 26 & 8 & 18 & \\
\hline \multicolumn{5}{|l|}{ Grade } \\
\hline G1 & 49 & 18 & 31 & \multirow[t]{2}{*}{0.9856} \\
\hline G2-G3 & 103 & 38 & 65 & \\
\hline \multicolumn{5}{|c|}{ Myometrial invasion (depth) } \\
\hline$\leq 1 / 2$ myometrium & 48 & 35 & 13 & \multirow[t]{2}{*}{$<0.0001^{\mathrm{a}}$} \\
\hline$>1 / 2$ myometrium & 104 & 21 & 83 & \\
\hline \multicolumn{5}{|l|}{ Nodal metastasis } \\
\hline Positive & 22 & 7 & 15 & \multirow[t]{2}{*}{0.5990} \\
\hline Negative & 130 & 49 & 81 & \\
\hline
\end{tabular}

ap $<0.05$, determined using the $\chi^{2}$ test. HS, histological score; PTGES2, prostaglandin E synthase 2; FIGO, International Federation of Gynecology and Obstetrics.
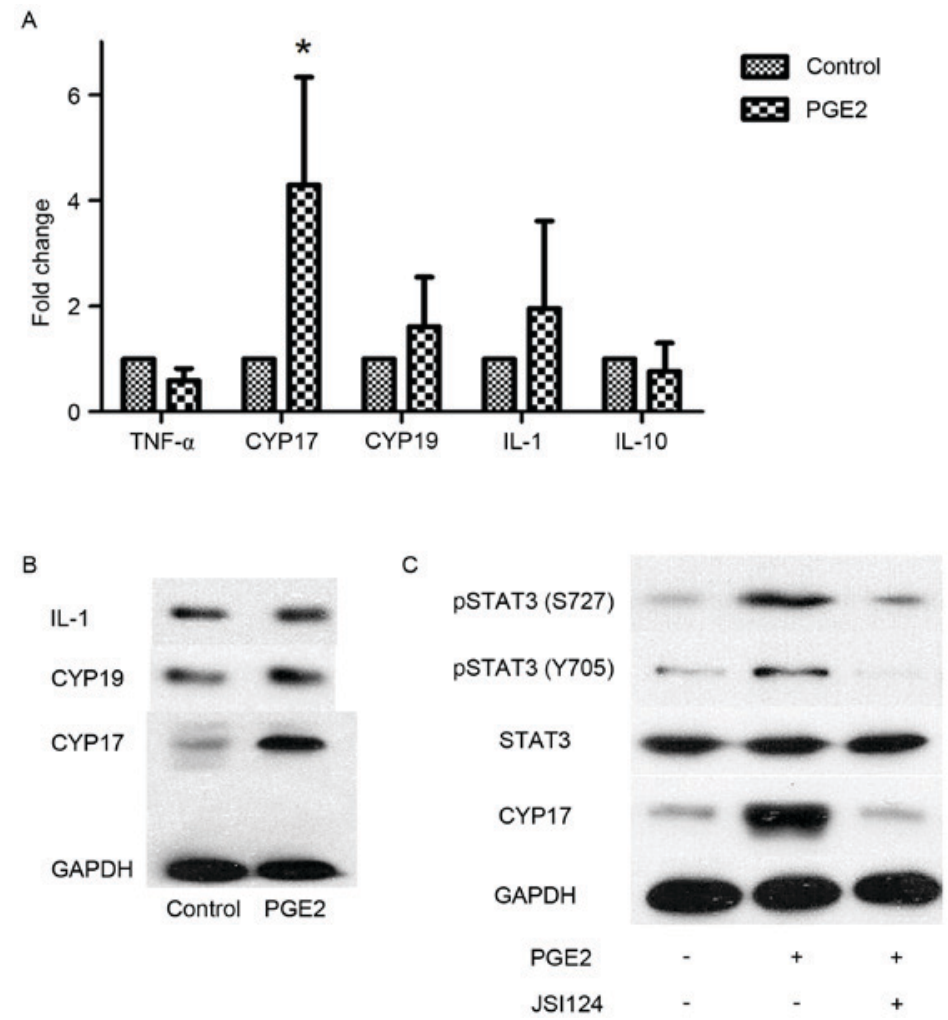

Figure 2. PGE2 promotes CYP17 expression in endometrial cancer cells via STAT3. (A) Reverse transcription-quantitative polymerase chain reaction analysis for TNF- $\alpha$, CYP17, CYP19, IL-1 and IL-10 in Ishikawa cells following stimulation with PGE2 (1x10 ${ }^{-9}$ mol/1). *P<0.05, analyzed by one-way analysis of variance. (B) Western blot analysis for IL-1, CYP19 and CYP17 in Ishikawa cells following stimulation with PGE2 (1x10 ${ }^{-9}$ mol/). (C) Western blot analysis for pSTAT3 S727, pSTAT3 Y705 and CYP17 in Ishikawa cells following stimulation with PGE2 (1x10 ${ }^{-9}$ mol/1) with or without JSI-124 inhibition. PGE2, prostaglandin E2; CYP, cytochrome P450; STAT3, signal transducer and activator of transcription 3; TNF- $\alpha$, tumor necrosis factor $\alpha$; IL, interleukin; PGE2, prostaglandin E2; pSTAT3, phosphorylated STAT3. 
A

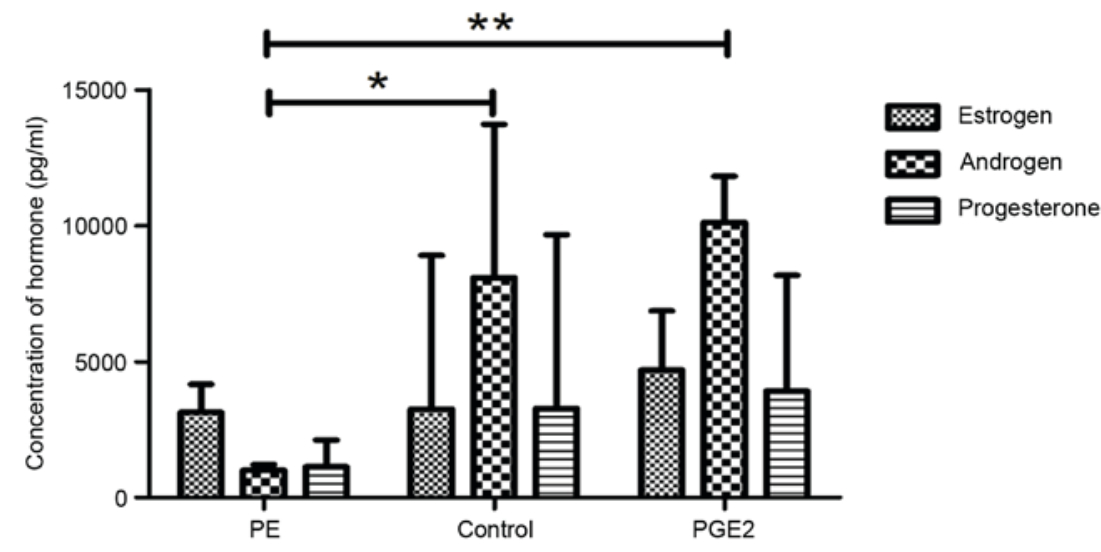

B

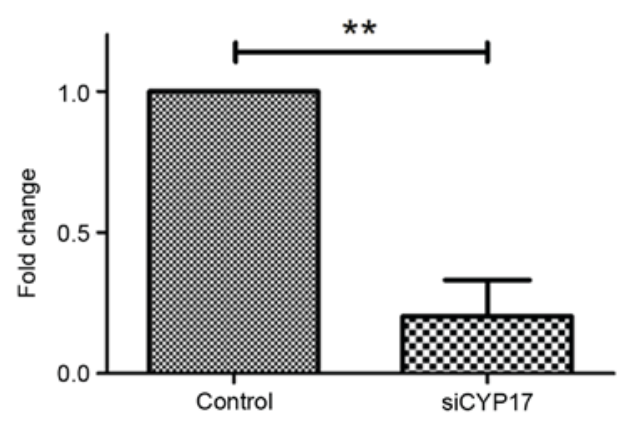

c

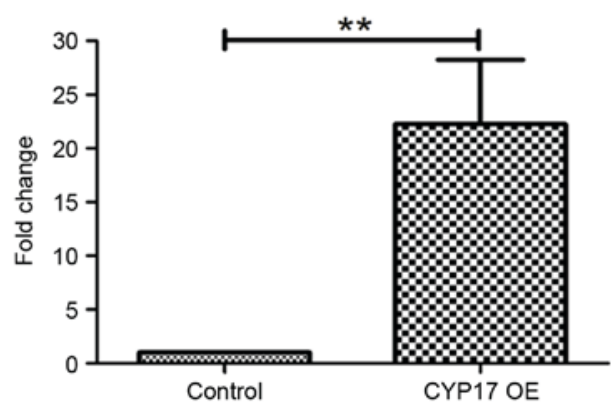

D

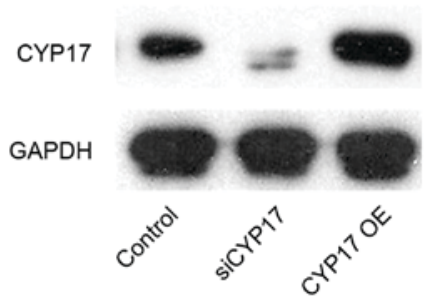

$\mathrm{E}$

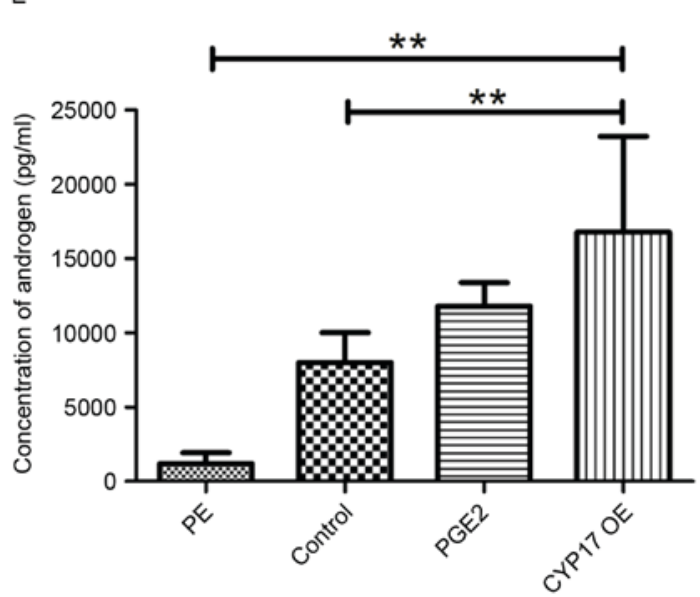

Figure 3. High expression of CYP17 promotes the secretion of androgens. (A) ELISA for estrogen, androgen and progesterone concentrations in PE cells, Ishikawa cells (control) and PGE2-stimulated Ishikawa cells. All cells were cultured for $48 \mathrm{~h}$. Reverse transcription-quantitative polymerase chain reaction analysis for Ishikawa cells (B) following transfection of siCYP17 and (C) in the CYP17 OE group. (D) Western blot analysis of Ishikawa cells in control groups, siCYP17 groups and CYP17 OE groups. (E) ELISA was performed to determine the concentration of androgens in PE cells, Ishikawa cells (control), PGE2-stimulated Ishikawa cells and CYP17 OE Ishikawa cells. All cells were cultured for $48 \mathrm{~h}$. "P<0.05, ${ }^{* *} \mathrm{P}<0.01$. CYP17, cytochrome P450 17 $\alpha$ hydroxylase; PE, primary endothelial; siCYP17, CYP17 small interfering RNA; CYP17 OE, CYP17 overexpression.

invasion was evaluated using a Transwell assay, the results of which indicated that siRNA Ishikawa cells had significantly lost their invasion ability when compared with the original Ishikawa cells following PGE2 stimulation (Fig. 4A and B). Ishikawa cells were classified as follows: The control group, the low androgen group (treated with $10^{-7} \mathrm{~g} / \mathrm{l}$ androgen), the high androgen group (treated with $10^{-5} \mathrm{~g} / \mathrm{l}$ androgen) and the CYP17 OE group. The Transwell results demonstrated that, compared with the control groups, invasion in the high androgen group and the CYP17 overexpression group was significantly increased (Fig. 4C and D). This suggests that CYP17 overexpression and a high concentration of androgen promotes invasion of endometrial cancer cells, initiated by PGE2 stimulation. 
A

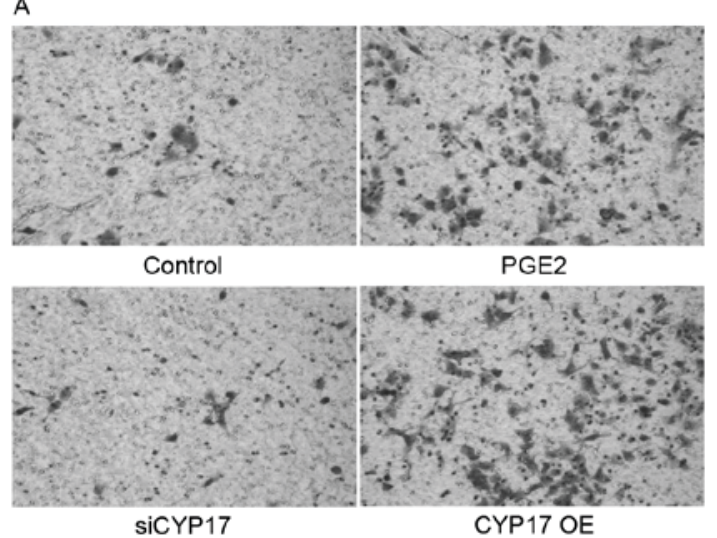

C

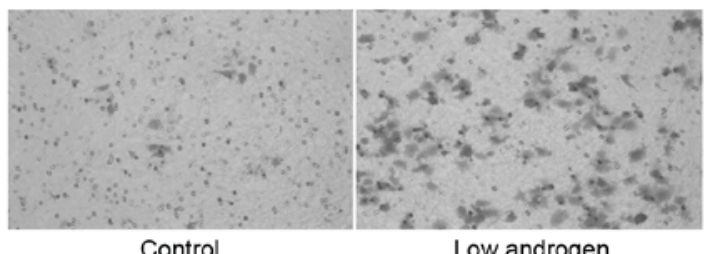

Control

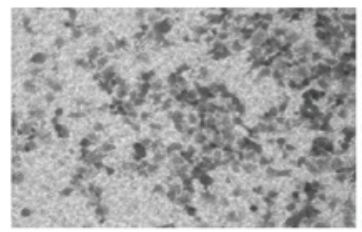

High androgen
Low androgen

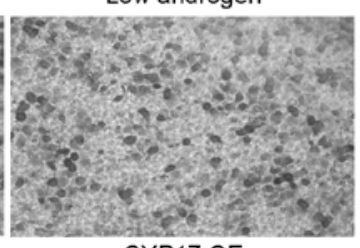

B

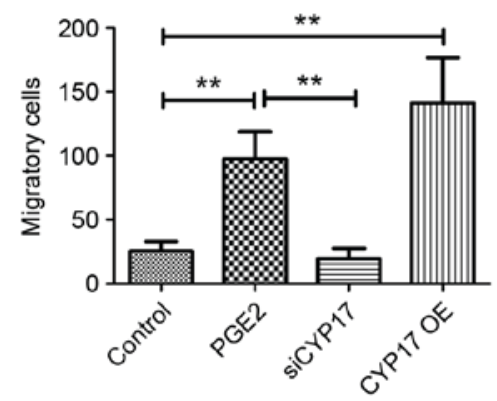

D

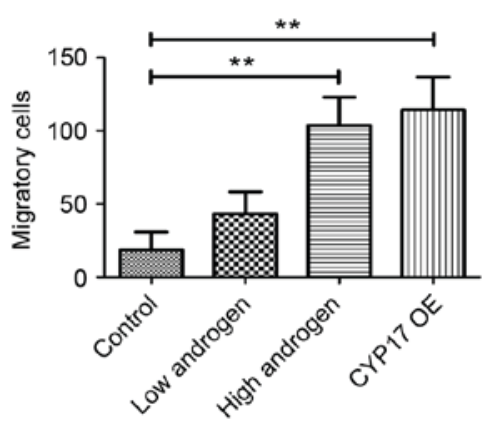

Figure 4. High expression of CYP17 promotes invasion in endometrial cancer cells. (A) Transwell migration assay images of Ishikawa cells in control, PGE2-stimulated, siCYP17 and CYP17 OE groups. Cells were stained with crystal violet. (B) The number of Ishikawa cells in control, PGE2 stimulated, siCYP17 and CYP17 OE groups (averaged across 5 random images). (C) Transwell migration assay images of Ishikawa cells in control, low androgen (treated with $10^{-7} \mathrm{~g} / 1$ androgen for $48 \mathrm{~h}$ ), high androgen (treated with $10^{-5} \mathrm{~g} / 1$ androgen for $48 \mathrm{~h}$ ) and CYP17 OE groups. Cells were stained with crystal violet. (D) The number of Ishikawa cells in control, low androgen, high androgen and CYP17 OE groups (averaged across five random images). ${ }^{* *} \mathrm{P}<0.01$, analyzed by one-way analysis of variance. Micrographs were taken at x200 magnification. CYP17, cytochrome P450 17 $\alpha$ hydroxylase; PGE2, prostaglandin E2; siCYP17, CYP17 small interfering RNA; CYP17 OE, CYP17 overexpression.

\section{Discussion}

The mediators and cellular effectors of inflammation are vital components of the local tumor microenvironment (29). Inflammation that promotes tumor development is an acknowledged enabling hallmark of cancer (30). PGE2 is an important inflammatory factor that acts as a tumor promoter in several cancer types $(31,32)$. In the present study, PTGES2, the synthase of PGE2, was revealed to be highly expressed in endometrial cancer cells, resulting in high expression of PGE2 in the microenvironment of endometrial cancer cells. As demonstrated in previous studies $(28,32)$, EP4 is the key PGE2 receptor in endometrial cancer, but the details of its function in cancer cells are complex and remain unclear (28). In the present study, PGE2 promoted CYP17 expression in endometrial cancer cells via STAT3.

The results of the present study indicated that CYP17 was more highly expressed following PGE2 stimulation. PGE2 is known to serve a role in endometrial cancer cells through its receptor, EP4 (28). With this in mind, in the present study, it was subsequently observed that STAT3 phosphorylation was increased with increased CYP17 expression. Therefore, STAT3 may promote CYP17 expression, via EP4, following
PGE2 stimulation in endometrial cancer cells. Subsequently, the concentration of androgens in endometrial cancer cells was determined and was revealed to be increased following CYP17 overexpression. As a result, invasion of endometrial cancer cells increased, accompanying cell behavior changes.

The cytochrome P450 family 17, subfamily A, member 1 gene provides instructions for making a member of the CYP enzyme family. Similar to other CYP enzymes, CYP17 is involved in the synthesis of steroid hormones (33). This group of hormones includes sex hormones, such as androgen and estrogen, which are required for normal sexual development and reproduction. The CYP17 enzyme performs two important reactions in this process, and existing evidence indicates that unopposed androgens contribute to the tumorigenesis and promotion of endometrial cancer $(34,35)$. Patients with polycystic ovary syndrome, who usually have high androgen levels, have an increased risk of developing endometrial cancer $(35,36)$. The results of the present study support this association and propose a potential mechanism that may begin with inflammation in the endometrium. Inflammation of the endometrium increased PGE2 levels in the microenvironment and resulted in increased CYP17 expression in endometrial cells and a higher concentration of 
total androgen in the extracellular environment. This change further results in tumorigenesis and invasion of endometrial cancer cells.

With regards to the mechanism by which PGE2 acts upon cellular gene expression, the present study observed that STAT3 serves an important role in promoting CYP17 expression in endometrial cancer cells. Previous studies have demonstrated that the STAT3 signaling pathway is persistently activated in tumors $(37,38)$. pSTAT3 forms homodimers or heterodimers, translocates into the nucleus and transactivates the expression levels of cyclin D1, vascular endothelial growth factor and matrix metalloproteinases-2/-9 genes. The protein products of these genes are involved in the regulation of tumor cell growth, apoptosis, angiogenesis and metastasis (39-42). It was clearly demonstrated that STAT3 is activated in endometrial cancer cells (43), and that STAT3 can stimulate PGE2 production (44). The results of the present study support a role for STAT3 in endometrial cancer, suggesting that a STAT3 inhibitor may be beneficial in the treatment of advanced-stage endometrial cancer when combined with PGE2/EP4 agonists.

In summary, the present study demonstrated a probable mechanism of invasion in endometrial cancer cells. The high expression of PGE2 in the microenvironment of endometrial cancer cells, possibly caused by inflammation, results in CYP17 overexpression in endometrial cancer cells via STAT3. Overexpression of CYP17 results in increased synthesis of androgens, which increases invasion of endometrial cancer cells. These findings also suggest that inflammation in the tumor microenvironment and high androgen levels serve important roles in tumorigenesis and invasion.

\section{Acknowledgements}

Not applicable.

\section{Funding}

The work was supported by Anhui Provincial Natural Science Foundation (grant no. 1808085QH274).

\section{Availability of data and materials}

The datasets generated and analyzed in the present study are included in the published article.

\section{Authors' contributions}

JK and DW conceived and designed the study. ZS, ML, CP, $\mathrm{PX}$ and $\mathrm{YZ}$ performed the experiments. MW provided the mutants. JK wrote the paper. XZ reviewed and edited the manuscript, and performed part of experiments such as IHC. JK reviewed and edited the manuscript. All authors read and approved the manuscript.

\section{Ethics approval and consent to participate}

Ethical approval was given by the medical ethics committee of Anhui Provincial Hospital. All patients provided consent to participate in this research.

\section{Patient consent for publication}

Patients in the manuscript provided written informed consent for the publication of any associated data and accompanying images.

\section{Competing interests}

The authors declare that they have no competing interests.

\section{References}

1. Jemal A, Bray F, Center MM, Ferlay J, Ward E and Forman D: Global cancer statistics. CA Cancer J Clin 61: 69-90, 2011.

2. Chen W, Zheng R, Zeng H, Zhang S and He J: Annual report on status of cancer in China, 2011. Chin J Cancer Res 27: 2-12, 2015.

3. Torre LA, Bray F, Siegel RL, Ferlay J, Lortet-Tieulent J and Jemal A: Global cancer statistics, 2012. CA Cancer J Clin 65: 87-108, 2015

4. von Euler US: On the specific vaso-dilating and plain muscle stimulating substances from accessory genital glands in man and certain animals (prostaglandin and vesiglandin). J Physiol 88: 213-234, 1936.

5. Legler DF, Bruckner M, Uetz-von Allmen E and Krause P: Prostaglandin E2 at new glance: Novel insights in functional diversity offer therapeutic chances. Int J Biochem Cell Biol 42. 198-201, 2010.

6. Che Q, Liu BY, Liao Y, Zhang HJ, Yang TT, He YY, Xia YH, $\mathrm{Lu} \mathrm{W}, \mathrm{He} \mathrm{XY}$, Chen Z, et al: Activation of a positive feedback loop involving IL- 6 and aromatase promotes intratumoral $17 \beta$-estradiol biosynthesis in endometrial carcinoma microenvironment. Int J Cancer 135: 282-294, 2014.

7. Zhu M, Che Q, Liao Y, Wang H, Wang J, Chen Z, Wang F, Dai C and Wan X: Oncostatin M activates STAT3 to promote endometrial cancer invasion and angiogenesis. Oncol Rep 34: 129-138, 2015.

8. Doherty GA, Byrne SM, Molloy ES, Malhotra V, Austin SC, Kay EW, Murray FE and Fitzgerald DJ: Proneoplastic effects of PGE2 mediated by EP4 receptor in colorectal cancer. BMC Cancer 9: 207, 2009 .

9. Frasor J, Weaver AE, Pradhan M and Mehta K: Synergistic up-regulation of prostaglandin E synthase expression in breast cancer cells by 17 beta-estradiol and proinflammatory cytokines. Endocrinology 149: 6272-6279, 2008.

10. Rask K, Zhu Y, Wang W, Hedin L and Sundfeldt K: Ovarian epithelial cancer: A role for PGE2-synthesis and signalling in malignant transformation and progression. Mol Cancer 5: 62, 2006.

11. Yu H, Lee H, Herrmann A, Buettner R and Jove R: Revisiting STAT3 signalling in cancer: New and unexpected biological functions. Nat Rev Cancer 14: 736-746, 2014.

12. Frank DA: STAT3 as a central mediator of neoplastic cellular transformation. Cancer Lett 251: 199-210, 2007.

13. Chaluvally-Raghavan P, Jeong KJ, Pradeep S, Silva AM, Yu S, Liu W, Moss T, Rodriguez-Aguayo C, Zhang D, Ram P, et al: Direct upregulation of STAT3 by MicroRNA-551b-3p deregulates growth and metastasis of ovarian cancer. Cell Rep 15: 1493-1504, 2016.

14. Banerjee $\mathrm{K}$ and Resat H: Constitutive activation of STAT3 in breast cancer cells: A review. Int J Cancer 138: 2570-2578, 2016.

15. Mora LB, Buettner R, Seigne J, Diaz J, Ahmad N, Garcia R, Bowman T, Falcone R, Fairclough R, Cantor A, et al: Constitutive activation of Stat 3 in human prostate tumors and cell lines: Direct inhibition of Stat 3 signaling induces apoptosis of prostate cancer cells. Cancer Res 62: 6659-6666, 2002.

16. Luwor RB, Stylli SS and Kaye AH: The role of Stat 3 in glioblastoma multiforme. J Clin Neurosci 20: 907-911, 2013.

17. Pilati $\mathrm{C}$ and Zucman-Rossi J: Mutations leading to constitutive active gp130/JAK1/STAT3 pathway. Cytokine Growth Factor Rev 26: 499-506, 2015.

18. Guengerich FP: Cytochrome p450 and chemical toxicology. Chem Res Toxicol 21: 70-83, 2008.

19. Vasaitis TS, Bruno RD and Njar VC: CYP17 inhibitors for prostate cancer therapy. J Steroid Biochem Mol Biol 125: 23-31, 2011.

20. Alex AB, Pal SK and Agarwal N: CYP17 inhibitors in prostate cancer: Latest evidence and clinical potential. Ther Adv Med Oncol 8: 267-275, 2016. 
21. Koh WJ, Greer BE, Abu-Rustum NR, Apte SM, Campos SM, Chan J, Cho KR, Cohn D, Crispens MA, Dupont N, et al: Uterine neoplasms, version 1.2014. J Natl Compr Canc Netw 12: 248-280, 2014.

22. Livak KJ and Schmittgen TD: Analysis of relative gene expression data using real-time quantitative PCR and the 2(-Delta Delta C(T)) method. Methods 25: 402-408, 2001.

23. Venturin GL, Chiku VM, Silva KL, de Almeida BF and de Lima VM: M1 polarization and the effect of PGE2 on TNFproduction by lymph node cells from dogs with visceral leishmaniasis. Parasite Immunol 38: 698-704, 2016.

24. Prosperi JR and Robertson FM: Cyclooxygenase-2 directly regulates gene expression of P450 Cyp19 aromatase promoter regions pII, pI.3 and pI.7 and estradiol production in human breast tumor cells. Prostaglandins Other Lipid Mediat 81: $55-70,2006$

25. Huang X, Jin J, Shen S, Xia Y, Xu P, Zou X, Wang H, Yi L, Wang Y and Gao Q: Modulation of expression of 17-Hydroxylase/17,20 lyase (CYP17) and P450 aromatase (CYP19) by inhibition of MEK1 in a human ovarian granulosa-like tumor cell line. Gynecol Endocrinol 32: 201-205, 2016.

26. Ramalho TR, Filgueiras LR, Pacheco de Oliveira MT, Lima AL, Bezerra-Santos CR, Jancar S and Piuvezam MR: Gamma-terpinene modulation of LPS-stimulated macrophages is dependent on the PGE2/IL-10 axis. Planta Med 82: 1341-1345, 2016.

27. Machado-Carvalho L, Martín M, Torres R, Gabasa M, Alobid I, Mullol J, Pujols L, Roca-Ferrer J and Picado C: Low E-prostanoid 2 receptor levels and deficient induction of the IL-1 $\beta /$ IL-1 type I receptor/COX-2 pathway: Vicious circle in patients with aspirin-exacerbated respiratory disease. J Allergy Clin Immunol 137: 99-107.e7, 2016.

28. Ke J, Yang Y, Che Q, Jiang F, Wang H, Chen Z, Zhu M, Tong H, Zhang H, Yan X, et al: Prostaglandin E2 (PGE2) promotes proliferation and invasion by enhancing SUMO-1 activity via EP4 receptor in endometrial cancer. Tumour Biol 37: 12203-12211, 2016.

29. Mantovani A, Allavena P, Sica A and Balkwill F: Cancer-related inflammation. Nature 454: 436-444, 2008.

30. Hanahan D and Weinberg RA: Hallmarks of cancer: The next generation. Cell 144: 646-674, 2011.

31. Kim JI, Lakshmikanthan V, Frilot $\mathrm{N}$ and Daaka Y: Prostaglandin E2 promotes lung cancer cell migration via EP4-betaArrestin1-c-Src signalsome. Mol Cancer Res 8: 569-577, 2010

32. Li S, Xu X, Jiang M, Bi Y, Xu J and Han M: Lipopolysaccharide induces inflammation and facilitates lung metastasis in a breast cancer model via the prostaglandin E2-EP2 pathway. Mol Med Rep 11: 4454-4462, 2015

33. Miller WR, Anderson TJ and Jack WJ: Relationship between tumour aromatase activity, tumour characteristics and response to therapy. J Steroid Biochem Mol Biol 37: 1055-1059, 1990.
34. Ito K, Miki Y, Suzuki T, McNamara KM and Sasano H: In situ androgen and estrogen biosynthesis in endometrial cancer: Focus on androgen actions and intratumoral production. Endocr Relat Cancer 23: R323-R335, 2016.

35. Goodman NF, Cobin RH, Futterweit W, Glueck JS, Legro RS and Carmina E; American Association of Clinical Endocrinologists (AACE); American College of Endocrinology (ACE); Androgen Excess and PCOS Society (AES): American association of clinical endocrinologists, american college of endocrinology and androgen excess and pcos society disease state clinical review: Guide to the best practices in the evaluation and treatment of polycystic ovary syndrome-part 1. Endocr Pract 21: 1291-1300, 2015.

36. Shao R, Li X and Billig H: Promising clinical practices of metformin in women with PCOS and early-stage endometrial cancer. BBA Clin 2: 7-9, 2014.

37. Gao SP, Mark KG, Leslie K, Pao W, Motoi N, Gerald WL, Travis WD, Bornmann W, Veach D, Clarkson B and Bromberg JF: Mutations in the EGFR kinase domain mediate STAT3 activation via IL-6 production in human lung adenocarcinomas. J Clin Invest 117: 3846-3856, 2007.

38. Berishaj M, Gao SP, Ahmed S, Leslie K, Al-Ahmadie H, Gerald WL, Bornmann W and Bromberg JF: Stat3 is tyrosine-phosphorylated through the interleukin-6/glycoprotein 130/Janus kinase pathway in breast cancer. Breast Cancer Res 9: R32, 2007.

39. Sai K, Wang S, Balasubramaniyan V, Conrad C, Lang FF, Aldape K, Szymanski S, Fokt I, Dasgupta A, Madden T, et al: Induction of cell-cycle arrest and apoptosis in glioblastoma stem-like cells by WP1193, a novel small molecule inhibitor of the JAK2/STAT3 pathway. J Neurooncol 107: 487-501, 2012.

40. Okazaki H, Tokumaru S, Hanakawa Y, Shiraishi K, Shirakata Y, Dai X, Yang L, Tohyama M, Hashimoto K and Sayama K: Nuclear translocation of phosphorylated STAT3 regulates VEGF-A-induced lymphatic endothelial cell migration and tube formation. Biochem Biophys Res Commun 412: 441-445, 2011.

41. Xie TX, Wei D, Liu M, Gao AC, Ali-Osman F, Sawaya R and Huang S: Stat 3 activation regulates the expression of matrix metalloproteinase-2 and tumor invasion and metastasis. Oncogene 23: 3550-3560, 2004.

42. Song Y, Qian L, Song S, Chen L, Zhang Y, Yuan G, Zhang H, Xia Q, Hu M, Yu M, et al: Fra-1 and Stat3 synergistically regulate activation of human MMP-9 gene. Mol Immunol 45: $137-143,2008$.

43. Subramaniam KS, Omar IS, Kwong SC, Mohamed Z, Woo YL, Mat Adenan NA and Chung I: Cancer-associated fibroblasts promote endometrial cancer growth via activation of interleukin-6/STAT-3/c-Myc pathway. Am J Cancer Res 6: 200-213, 2016.

44. Gao J, Tian J, Lv Y, Shi F, Kong F, Shi H and Zhao L: Leptin induces functional activation of cyclooxygenase-2 through JAK2/STAT3, MAPK/ERK, and PI3K/AKT pathways in human endometrial cancer cells. Cancer Sci 100: 389-395, 2009. 